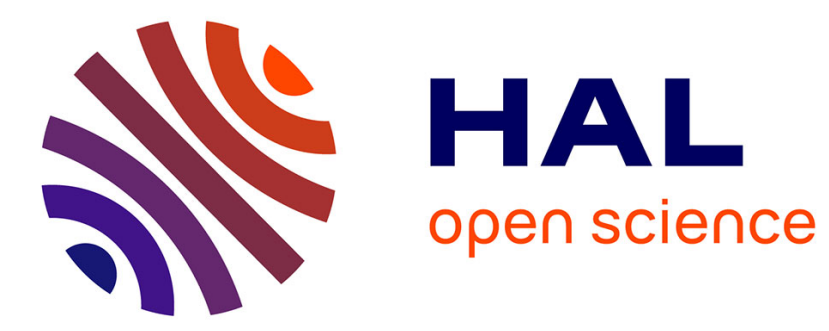

\title{
Different Approaches used in Modeling of Cell Membrane Electroporation
}

Clair Poignard, A Silve, L Wegner

\section{To cite this version:}

Clair Poignard, A Silve, L Wegner. Different Approaches used in Modeling of Cell Membrane Electroporation. [Research Report] RR-8940, Inria Bordeaux Sud-Ouest. 2016. hal-01349523v2

\section{HAL Id: hal-01349523 \\ https://hal.inria.fr/hal-01349523v2}

Submitted on 9 Feb 2017

HAL is a multi-disciplinary open access archive for the deposit and dissemination of scientific research documents, whether they are published or not. The documents may come from teaching and research institutions in France or abroad, or from public or private research centers.
L'archive ouverte pluridisciplinaire HAL, est destinée au dépôt et à la diffusion de documents scientifiques de niveau recherche, publiés ou non, émanant des établissements d'enseignement et de recherche français ou étrangers, des laboratoires publics ou privés. 
Different Approaches used in Modeling of Cell Membrane Electroporation.

C. Poignard, A. Silve, L. Wegner

RESEARCH

REPORT

$\mathbf{N}^{\circ} 8940$

July 2016

Project-Team MONC 



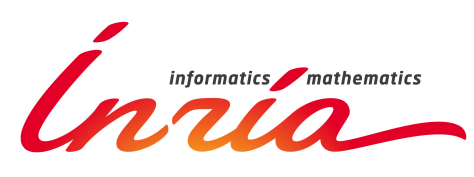

\title{
Different Approaches used in Modeling of Cell Membrane Electroporation.
}

\author{
C. Poignard, A. Silve, L. Wegner ${ }^{\dagger}$ \\ Project-Team MONC
}

Research Report n 8940 - July 2016 - 21 pages

\begin{abstract}
Cell electroporation is a complex phenomenon, which consists in the emergence of defects in cell membranes subjected to electric pulses. Since the end of the 90's biophysical models have been developed to explain and predict the conditions for cell electroporation. However the recent biological data, in particular those dealing with the influence of the repetition rate of the pulses challenge these biophysical models. In this chapter we introduce different biophysical models of electropore formation and we discuss their mathematical basis and their advantages and disadvantages. We also present the phenomenological modeling, which consists in designing the model on an empirical basis thanks to the experience. The aim of the chapter is to introduce the reader to different ways of modeling cell membrane electroporation, and to provide some possible directions to obtain a more reliable theory of electroporation in accordance with the experiments and with a justified theoretical basis.
\end{abstract}

Key-words: Cell electroporation modeling, biophysical models for pore formation, mathematical models for membrane electroporation

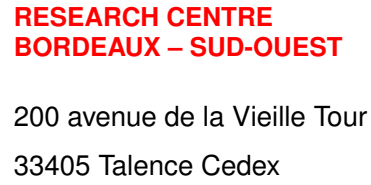




\section{Différentes approches pour la modélisation de l'électroporation des membranes cellulaires.}

Résumé : Le but de ce rapport est de présenter les différentes approches biophysiques et phénoménologiques développées pour décrire l'électroperméabilisation de la membrane cellulaire. Nous repartons des modèles simples de circuits électriques équivalents, et celui de Schwan décrivant la membrane comme un condensateur mis en parallèle avec une résistance. Ensuite nous présentons les principes de la théorie de l'électroporation développée dans les années 90 ' à partir des énergies libres des membranes, et qui a fait l'objet depuis de quelques modifications. Enfin nous présentons les approches phénoménologiques récentes qui permettent de comparer les données expérimentales et les simulations numériques. L'objectif est présenter les avantages et la désavantages de chaque approche afin d'ouvrir de possibles directions de recherches pour obtenir une théorie de l'électroporation plus en accord avec les observations, tout en se fondant sur une base théorique solide

Mots-clés : Modélisation de l'électroporation cellulaire, modèles biophysiques pour la formation de pores, modèles mathématiques pour l'électroporation des membranes cellulaires 


\section{Contents}

1. Introduction 4

2. Cell electrical modeling before electroporation: the linear regime 5

$\begin{array}{lll}2.1 . & \text { Equivalent circuit for cell: the 0D-model } & 5\end{array}$

2.2. 3D Maxwell equations and their simplication to the 3D-model 6

\begin{tabular}{lll}
\hline 2.3. & Spherical cell in a unidirectional constant field & 7
\end{tabular}

$3 . \quad$ Basic concepts in biophysics for pore formation in liposomes 9

$\begin{array}{lll}3.1 . & \text { Single pore models } & 9\end{array}$

$\begin{array}{lll}3.2 . & \text { From one pore to the total pore density equation } & 10\end{array}$

$\begin{array}{lll}3.3 . & \text { Pore radii evolution } & 11\end{array}$

\begin{tabular}{lll}
\hline 3.4. & The KN-model for electroporation current & 11
\end{tabular}

3.5. Back to the electric equation and drawbacks of the KN-model 12

$\begin{array}{lll}4 . & \text { Phenomenological models for membrane conductivity } & 14\end{array}$

\begin{tabular}{lll}
\hline 4.1. & Biological evidences of electroporation & 15
\end{tabular}

4.2. The two-step process of membrane electroporation 15

\begin{tabular}{|lll}
\hline 4.3. & Calibration of the model with patch-clamp experiments & 16
\end{tabular}

\begin{tabular}{|lll}
\hline $4.4 . \quad$ Biophysical basis of the phenomenological and link with pore radius evolution & 17
\end{tabular}

\begin{tabular}{|lr}
\hline 4.5. & The role of the surface diffusion \\
\hline
\end{tabular}

\begin{tabular}{|lr}
$5 . \quad$ Concluding remarks and perspectives & 18
\end{tabular}

\begin{tabular}{ll}
\hline Acknowledgements & 20
\end{tabular}

\begin{tabular}{lr}
\hline References & 20
\end{tabular} 


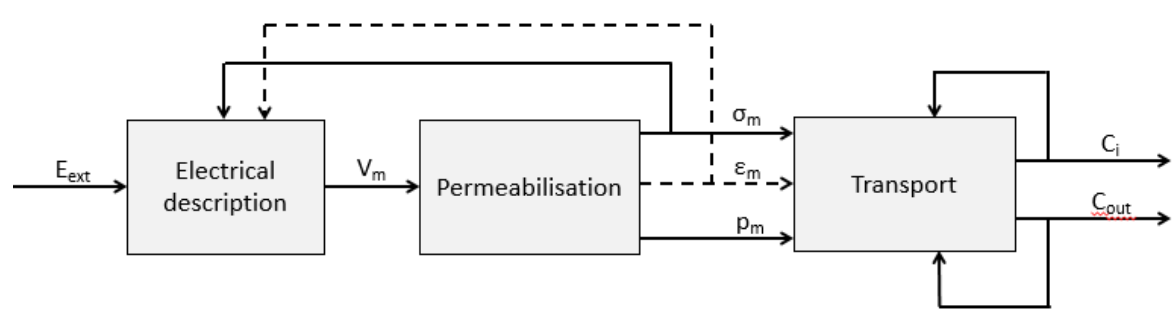

Figure 1. Schematic process of electroporation.

\section{INTRODUCTION}

Eukaryotic cell is a complex biological entity, which is the main constituent of any biological tissues: it is somehow the base unit of any living organism. These cells are generically composed of cytoplasm, which includes nucleus, mitochondria and other organelles that are necessary for life. This cytoplasm is protected from the extracellular stress by the plasma membrane, which is a phospholipid bilayer. This barrier plays a double role of protecting the cell and controlling exchanges between the cytoplasm and the extracellular medium. In the 70's, it has been observed that electric shock may change transiently the membrane, allowing the entrance of usually non-permeant molecules into the cytoplasm. This phenomenon, called electroporation or electropermeabilization has then been studied for cancer treatments, by treating cells with cytototoxic drugs - such as bleomycin or cisplatin - while exposing them to pulsed electric fields. This treatment called electrochemotherapy (see the chapters by L.M Mir, Gehl and Serša, and also Cadossi et al.) is now used standardly in more than 40 Cancer Institutes in Europe for cutaneaous tumors and several clinical studies are driven for deep located tumors. Even though the bases of cell electroporation are well-known, several experimental observations are still unexplained and the modeling of the phenomenon suffers from a lack of accuracy. The aim of this chapter consists in presenting different approaches to model cell membrane electroporation, and in discussing the pros and cons of each approach.

Generally speaking, the process of electroporation can be schematized by Figure 1. Exposure to applied external electrical field leads to an increase of the transmembrane voltage $V_{\mathrm{m}}$, which induces an increase of membrane conductivity $\sigma_{\mathrm{m}}$, permittivity $\varepsilon_{\mathrm{m}}$ and permeability $p_{\mathrm{m}}$. Increased membrane conductivity and permittivity, in turn, affect membrane voltage til the end of the pulse. Membrane permeability is not intrinsic: it depends on the considered molecule, and is detected thanks to transport of molecules (PI, DNA plasmids...). The aim of the chapter is to focus on the electrical description of the cell membrane, without accounting for the transport process, which is still unclear. The chapter is organized into 5 sections. First, the linear electric model of Schwan et al. [5] is presented. The cell is composed of a conducting cytoplasm surrounded by a resistive thin layer. Equivalent circuit model is described and complexified by the generic model in which the thin membrane is accounted for by imposing equivalent transmission conditions across the interface between the cytoplasm and the outer medium. Electroporation phenomenon modeling is then addressed. In Section 3 the biophysical models for electropore 
formation are briefly introduced and the model of Krassowska and Neu referred to as the KNmodel, considered as the most advanced description of electroporation, is described. In Section 4 phenomenological approaches which consist in designing a model thanks to the experimental observations are proposed. Phenomenological models avoid the drawbacks of the other models, in particular when considering the effect of pulse repetition. In conclusion, current challenges in cell electroporation modeling are given, in order to obtain models reliable and in complete accordance with the experiments.

\section{Cell electrical modeling Before electroporation: the linear Regime}

In the Schwan model [5], the cell is composed of a homogeneous conducting cytoplasm, which is roughly tens of micrometers in diameter, surrounded by a few nanometers thick, insulating membrane (see Figure 2).

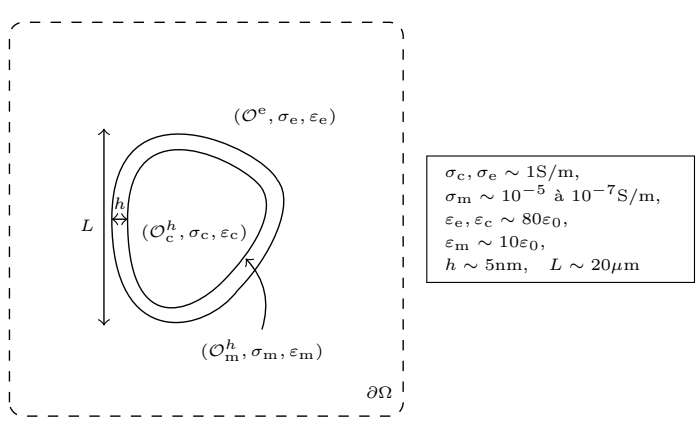

(a) Schematic cell and dielectric parameters
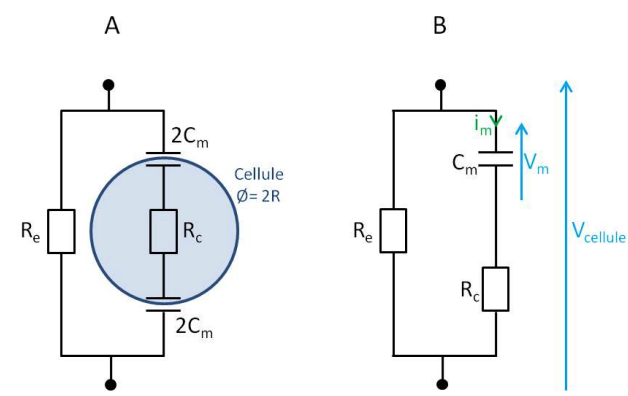

(b) Equivalent electric cell model

Figure 2. Electrical model of biological cell by Schwan, et al. [5 and its equivalent electric circuit. The cytoplasm $\mathcal{O}_{\mathrm{c}}$ is protected thanks to the thin membrane $\mathcal{O}_{\mathrm{m}}$, whose thickness $h$ is about a few nanometers. The cell is embedded in an extracellular medium denoted by $\mathcal{O}^{\mathrm{e}}$. The entire domain is denoted by $\Omega$.

2.1. Equivalent circuit for cell: the 0D- model. The simplest way to model the cell is to derive an electric circuit model in which the cell cytoplasm is described by a resistivity $R_{c}$, the cell membrane is identified by a capacitor whose capacitance equals $\mathrm{C}_{\mathrm{m}}$ and the ambient medium is described by a resistivity $\mathrm{R}_{\mathrm{e}}$ as given by Figure 2(b). Kirchhoff 's circuit law writes then

$$
V_{\text {cell }}=V_{\mathrm{m}}+\mathrm{R}_{\mathrm{c}} \mathrm{C}_{\mathrm{m}} \frac{d V_{\mathrm{m}}}{d t} .
$$

If a static electric field of magnitude $E$ is applied to the cell of radius $r_{\mathrm{c}}$ one infers

$$
2 r_{\mathrm{c}} E=V_{\mathrm{m}}+\mathrm{R}_{\mathrm{c}} \mathrm{C}_{\mathrm{m}} \frac{d V_{\mathrm{m}}}{d t} .
$$

Assuming that $V_{\mathrm{m}}$ is zero at the initial time and that the electric field $E$ is constant, then $V_{\mathrm{m}}$ is given by

$$
V_{\mathrm{m}}(t)=2 r_{\mathrm{c}} E\left(1-e^{-t / \tau}\right), \quad \text { with } \quad \tau=\mathrm{R}_{\mathrm{c}} \mathrm{C}_{\mathrm{m}}
$$

RR n 8940 
Interestingly, this very simple model exhibits a linear dependency of the transmembrane voltage on the radius. Such a linear dependency is intrinsic and it will be recovered in the 3D-model, as explained below. However this equivalent model is too rough, and in particular it cannot describe the influence of the cell shape or the effect of the direction of the electric field on the membrane voltage. Therefore, it is necessary to use partial differential equations (PDEs), which describe the electric field in the whole cell.

2.2. 3D Maxwell equations and their simplication to the 3D-model. Maxwell equations and the standard constitutive laws for a dielectric material with permittivity $\varepsilon$ and conductivity $\sigma$ read

$$
\operatorname{curl} \mathbf{H}=\varepsilon \partial_{t} \mathbf{E}+\sigma \mathbf{E}, \quad \operatorname{curl} \mathbf{E}=-\operatorname{curl} \mathbf{H} .
$$

This 3D-vector system is quite complex to solve, especially for high contrast material as biological cells. However, neglecting the time-variation of the magnetic field leads to a curl-free electric field $\mathbf{E}$, which then implies that $\mathbf{E}$ derives from a scalar potential $\mathbf{E}=-\nabla V$. Then, taking the divergence of the left equation leads to the following PDE on $V$ called the electroquasistatic formulation:

$$
\nabla \cdot\left(\varepsilon \nabla \partial_{t} V\right)+\nabla \cdot(\sigma \nabla V)=0,
$$

to which initial and boundary conditions are imposed, for instance

$$
\left.V\right|_{\partial \Omega}=g,\left.\quad V\right|_{t=0}=V_{0} .
$$

For the cell cytoplasm and the extracellular medium, the ratio $\varepsilon / \sigma$ is about $10^{-9} s$ (see the parameters of Fig 2(a)], meaning that up to several mega-hertz, the displacement currents can be neglected. However, due to the high resistivity of the membrane, these currents have to be accounted for in the thin layer [16], hence $V$ is the continuous solution to

$$
\begin{aligned}
& \nabla \cdot(\sigma \nabla V)=0, \quad \text { in } \mathcal{O}_{\mathrm{c}}^{h} \cup \mathcal{O}_{\mathrm{m}}^{h} \cup \mathcal{O}^{\mathrm{e}}, \\
& \left.\sigma \partial_{\mathbf{n}} V\right|_{\Gamma^{+}, \Gamma_{h}^{-}}=\left.\varepsilon_{\mathrm{m}} \partial_{t} \partial_{\mathbf{n}} V\right|_{\Gamma^{-}, \Gamma_{h}^{+}}+\left.\sigma_{\mathrm{m}} \partial_{\mathbf{n}} V\right|_{\Gamma^{-}, \Gamma_{h}^{+}}, \quad \text { on } \Gamma \text { and } \Gamma_{h} \text { respectively, } \\
& \left.V\right|_{\partial \Omega}=g,\left.\quad V\right|_{t=0}=V_{0},
\end{aligned}
$$

where $\Gamma_{h}$ and $\Gamma$ are the respective outer and inner boundaries of the cell membrane, and the normal vectors are taken from the inner to the outer part of the cell. Even though this equation is a rough simplification of the Maxwell vector equations, $\nabla V$ describes quite precisely the electric field at low frequency and thus it is widely used in the electrical bioengineering community. However, due to the high resistivity and the small thickness of the membrane, it is still complex to solve accurately the above equation on $V$.

To perform computations on realistic cell shapes without meshing the cell membrane, Pucihar et al. [19] propose to replace the membrane by an equivalent condition on the boundary of the cytoplasm, see Figure 3 Denoting by $\mathrm{S}_{0}$ the surface conductance and by $\mathrm{C}_{\mathrm{m}}$ the capacitance of the membrane defined as

$$
\mathrm{C}_{\mathrm{m}}=\varepsilon_{\mathrm{m}} / h, \quad \mathrm{~S}_{0}=\sigma_{\mathrm{m}} / h,
$$




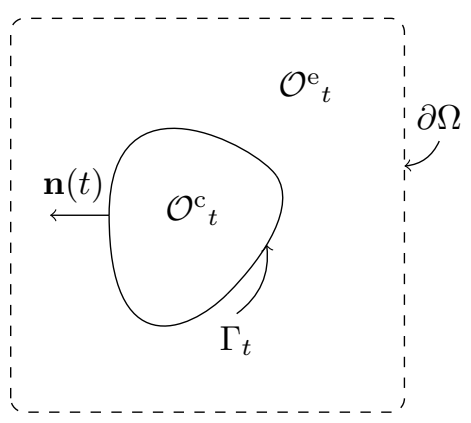

Figure 3. Cell model with a zero-thickness membrane. The influence of the membrane is described through its capacitance $\mathrm{C}_{\mathrm{m}}$ and its surface conductance $\mathrm{S}_{0}$.

and denoting by $\mathcal{O}_{\mathrm{c}}$ the whole cell

$$
\mathcal{O}_{\mathrm{c}}=\overline{\mathcal{O}_{\mathrm{c}}^{h}} \cup \mathcal{O}_{\mathrm{m}}
$$

the electric potential $V$ is approached by $U$, the solution to

$$
\begin{aligned}
& \Delta U=0, \quad \text { in } \mathcal{O}_{\mathrm{c}} \cup \mathcal{O}^{\mathrm{e}}, \\
& \left.\sigma_{\mathrm{e}} \partial_{\mathbf{n}} U\right|_{\Gamma^{+}}=\left.\sigma_{\mathrm{c}} \partial_{\mathbf{n}} U\right|_{\Gamma^{-}}, \\
& \mathrm{C}_{\mathrm{m}} \partial_{t} V_{\mathrm{m}}+\mathrm{S}_{0} V_{\mathrm{m}}=\left.\sigma_{\mathrm{c}} \partial_{\mathbf{n}} U\right|_{\Gamma^{-}}, \quad \text { where } V_{\mathrm{m}}=\left.U\right|_{\Gamma^{+}}-\left.U\right|_{\Gamma^{-}}, \\
& \left.U\right|_{\partial \Omega}=g,\left.\quad U\right|_{t=0}=V_{0} .
\end{aligned}
$$

It is worth noting that unlike $V$, the approximate potential $U$ is discontinuous across the interface: this is the effect of the high resistivity and the small thickness of the membrane. Equation (12c) corresponds to a contact resistance model, rigorously justified by Poignard and Perrussel[15]. It is a generalization of (2), accounting the cell geometry and the electric field orientation thanks to the Neumann derivative.

2.3. Spherical cell in a unidirectional constant field. For simple shapes and simple electric field, it is possible to compute analytically the transmembrane voltage. For instance, let assume that the cell is a sphere of radius $r_{\mathrm{c}}$ embedded in the whole space $\mathbb{R}^{3}$ and submitted to the unidirectional electric field $\mathbf{E}=E(t) \mathbf{e}_{\mathbf{z}}$. Using polar coordinates $-x=r \cos \theta \sin \varphi, y=$ $r \sin \theta \sin \varphi, z=r \cos \varphi$ - the Kirchoff law (12c) reads

$$
\mathrm{C}_{\mathrm{m}} \partial_{t} V_{\mathrm{m}}+\left(\mathrm{S}_{0}+\frac{2 \sigma_{\mathrm{e}} \sigma_{\mathrm{c}}}{r_{\mathrm{c}}\left(2 \sigma_{\mathrm{e}}+\sigma_{\mathrm{c}}\right)}\right) V_{\mathrm{m}}=\frac{3 \sigma_{\mathrm{e}} \sigma_{\mathrm{c}}}{2 \sigma_{\mathrm{e}}+\sigma_{\mathrm{c}}} E(t) \cos \varphi .
$$

Therefore, for a time-constant electric field one obtains the explicit expression of $V_{\mathrm{m}}$ :

$$
V_{\mathrm{m}}(t, \varphi)=\frac{3}{2} r_{\mathrm{c}} E \frac{1}{1+\frac{\sigma_{\mathrm{c}}+2 \sigma_{\mathrm{e}}}{2 \sigma_{\mathrm{c}} \sigma_{\mathrm{e}}} r_{\mathrm{c}} \mathrm{S}_{0}}\left(1-e^{-t / \tau_{\mathrm{m}}}\right) \cos \varphi, \quad \text { with } \quad \tau_{\mathrm{m}}=\frac{r_{\mathrm{c}} C_{\mathrm{m}}}{\frac{2 \sigma_{\mathrm{c}} \sigma_{\mathrm{e}}}{\sigma_{\mathrm{c}}+2 \sigma_{\mathrm{e}}}+r_{\mathrm{c}} \mathrm{S}_{0}}
$$

$\mathrm{RR} \mathrm{n}^{\circ} 8940$ 
In the linear regime, the membrane conductance is of order 1, and since the cell radius is of the order tens of microns, one has:

$$
\frac{1}{1+\frac{\sigma_{\mathrm{c}}+2 \sigma_{\mathrm{e}}}{2 \sigma_{\mathrm{c}} \sigma_{\mathrm{e}}} r_{\mathrm{c}} \mathrm{S}_{0}} \sim 1, \quad \text { and } \tau_{\mathrm{m}} \sim \frac{r_{\mathrm{c}} C_{\mathrm{m}}\left(\sigma_{\mathrm{c}}+2 \sigma_{\mathrm{e}}\right)}{2 \sigma_{\mathrm{c}} \sigma_{\mathrm{e}}} .
$$

Thus one recovers the linear dependency of the membrane voltage with respect to the cell radius, but with a different constant compared with the equivalent circuit model.

One interesting feature of formula (14) lies in the fact that the membrane conductance has to increase dramatically to impact the membrane voltage. Roughly, for values of $\mathrm{S}_{0}$ increasing from 1 to $10^{4} \mathrm{~S} / \mathrm{m}^{2}$, the changes on the potential is only about $10 \%$. Therefore direct measurements of the membrane potential cannot detect any increase of membrane conductance below $1000 \mathrm{~S} / \mathrm{m}^{2}$. Another interesting feature is that the constant time $\tau_{\mathrm{m}}$ is bounded by below by $r_{\mathrm{c}} C_{\mathrm{m}}\left(\sigma_{\mathrm{c}}+\right.$ $\left.2 \sigma_{\mathrm{e}}\right) /\left(2 \sigma_{\mathrm{c}} \sigma_{\mathrm{e}}\right)$, which is about several tenth of microseconds for cells, and thus the membrane voltage stabilizes within a few microseconds.

Formula (14) predicts the sinusoidal behavior of the transmembrane voltage in the linear regime as observed by the experimental data (Figure 4).
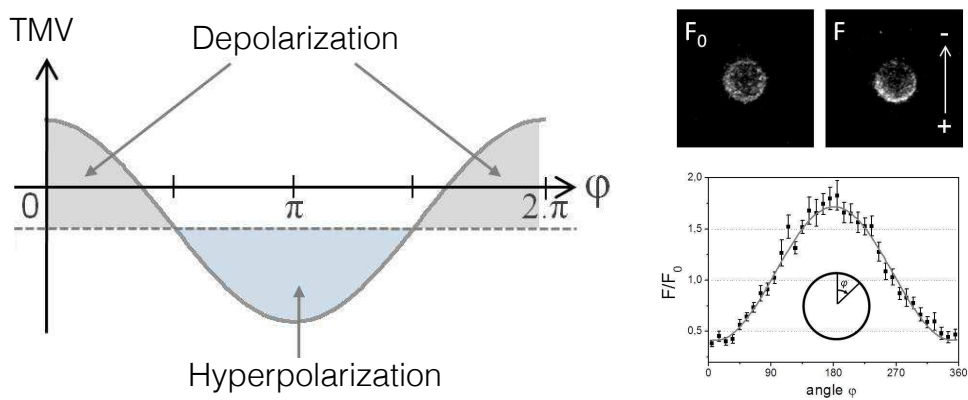

Figure 4. Left: Schematic angular variation of the transmembrane voltage according formula (14). Right: Fluorescent measurements, and relative fluorescent with respect to the angle. From [17].

Remark 1 (On the Scwhann equation and short pulses). As mention above, for square pulses longer than the intrisinc membrane charging time $\tau_{\mathrm{m}}$, equality (14) leads to the so-called Schwann equation, which gives the transmembrane voltage value at the end of the pulse: $V_{\mathrm{m}} \sim_{t \gg \tau_{\mathrm{m}}}$ $(3 / 2) r_{\mathrm{c}} E \cos \varphi$, since $\left(1-e^{-t / \tau_{m} r m}\right) \sim 1$. It is worth noting that such formula does not hold for short pulses, which lasts a few nanoseconds. For such pulses, the membrane voltage is approximated by

$$
V_{\mathrm{m}}(t, \varphi) \sim_{t \ll \tau_{\mathrm{m}}} \frac{3}{2} r_{\mathrm{c}} \frac{t}{\tau_{\mathrm{m}}} \mathcal{M}(E) \cos \varphi \sim \frac{3 \sigma_{\mathrm{e}} \sigma_{\mathrm{c}}}{\sigma_{\mathrm{c}}+2 \sigma_{\mathrm{e}}} \frac{t}{C_{\mathrm{m}}} \mathcal{M}(E) \cos \varphi,
$$

where $\mathcal{M}(E)$ is the mean value of $E$ along the pulse:

$$
\mathcal{M}(E)=\frac{1}{t} \int_{0}^{t} E(s) d s .
$$


The important fact is that the transmembrane voltage for short pulses does not depend on the cell radius, but on the time of pulse application and the mean value of the field amplitude is involved.

\section{BASIC CONCEPTS IN BIOPHYSICS FOR PORE FORMATION IN LIPOSOMES}

The creation and growth of single pores in vesicles have been studied for many years [31, 23, 10 , 22. At the end of the 90's, Sandre et al. 23. have studied the pore creation in vesicles embedded in a highly viscous fluid. The high viscosity of the ambient medium made possible to visualize pore formation in stretched vesicles in real time, and thus the comparison of the theory with the experiments. Unfortunately, such experiments have not been performed in electroporation research. Note that changes in the medium viscosity would imply dramatic changes in the medium conductivity and in the lipid properties, which thus would complexify the modeling. However since the end of the 90's, biophysicists proposed different model for pore formation in membranes subjected to high transmembrane voltage. An extensive and physical presentation of such approaches is given in the first section, Chapters 1 and 2 by J. Weaver.

3.1. Single pore models. Pore radius models are based on the description of the free-energy of membranes, thanks to which Langevin-type equation provides the pore radius evolution. More precisely, given the membrane energy $E_{m}$ as a function of the pore radius $r$, the time-evolution of the single pore radius $r$ behaves as follows accordingly Weaver and Chizmadzhev [31:

$$
\frac{d r}{d t}=-\frac{D}{k_{B} T} \partial_{r} E_{m}
$$

where $D, k_{B}$ and $T$ hold respectively for the diffusion coefficient, the Boltzmann constant and the temperature. The two next subsections deal with the two main models that describe the single pore radius, that referred to respectively as BGS-CW model and DAV-model.

3.1.a. The classical BGS-CW model. Brochart-Wyart, de Gennes and Sandre [23] on one side and Chizmadzhev and Weaver [31] on the other side proposed the membrane free-energy $E_{m}$ given by:

$$
E_{m}(r)=E_{f}(r)+E_{p}(r)=2 \pi \gamma r-\pi r^{2}\left(\sigma_{0}+a_{p} V_{\mathrm{m}}^{2}\right)+\frac{C_{s}}{r^{4}} .
$$

The theoretical basis of this energy is presented in J.C. Weaver's Chapter 1 "Electropore Energy and Thermodynamics". In the above equation, $\gamma$ is the line tension, which tends to shrink the pore, while $\sigma_{0}$ is the surface tension of the stretched vesicles. The term $C_{s}$ holds for the steric repulsion of the lipids. It ensures that a small space $r_{0}$ between the phospholipids remains at rest, and has a very low influence for pore radii above $r_{0}$. The term $a_{p}$ is the membrane capacitance and $V_{\mathrm{m}}$ is the transmembrane voltage. The pore radius evolution, which is called here $B G S-C W$ model, is then derived thanks to 18 and Stokes-Einstein -that is $k_{B} T / D=2 \pi \eta_{\mathrm{m}} \delta$ where $\delta$ is the membrane thickness- such that

$$
\frac{d r}{d t}=-\frac{1}{\eta_{\mathrm{m}} \delta}\left(\gamma-r\left(\sigma_{0}+a_{p} V_{\mathrm{m}}^{2}\right)-\frac{C_{s}}{r^{5}}\right) .
$$

One can point out a few drawbacks of the model. The first one lies in the fact that pores are assumed cylindrical all along their lives. Such assumption seems justified during the formation, $\mathrm{RR} \mathrm{n}^{\circ} 8940$ 
according to the very recent study of Sengel and Wallace [25]. However there is no physical justification of circular shrinkage, in particular the lateral diffusion of membrane lipids is important (a few $\mathrm{nm}^{2}$ per ns) and should change the shape of the defects. In addition pore expansion is exponentially fast above the critical radius $r_{c}$ given by

$$
r_{c} \sim \frac{\gamma}{\sigma_{0}+a_{p} V_{\mathrm{m}}^{2}},
$$

which is hardly defensible since the pore radius should be bounded at least by half of the membrane circumference. On the other hand, if the membrane voltage is stoppped, the pore shrinks, but the shrinkage is linear as soon as the radius is smaller than $\gamma / \sigma_{0}$, whereas Sandre et al. have reported an acceleration of the shrinkage for small radii[23]. More recently, Kroeger, Ryham, et al. [10, 22] have pointed out that experiments are at odds with the linear closure, and they derived in two different ways a curvature-driven pore model.

3.1.b. Curvature-driven pore closure: the role of aequous viscosity. In [22, Ryham et al. suggested that the aequous viscosity of the ambient medium impacts the pore dynamics. They derived the Dominant Aequous Viscosity (DAV) model by adding a force $F_{s}$, which accounts for the lateral stresses generated on the bilayer:

$$
F_{s}=C \eta_{s} r \frac{d r}{d r}
$$

where $C$ is a non-dimension constant, whose value is around $C \sim 8$ according to [22], and $\eta_{s}$ is the viscoity of the solution. Summing up all the forces, the following equation on the pore radius evolution in membrane submitted to a transmembrane voltage holds:

$$
C \eta_{s} r \frac{d r}{d t}+\eta_{\mathrm{m}} \delta \frac{d r}{d t}=-\gamma+r\left(\sigma_{0}+a_{p} V_{\mathrm{m}}^{2}\right)+\frac{C_{s}}{r^{5}} .
$$

Note that the steric repulsion $C_{s} / r^{5}$ is not given in the DAV model as written in [22], however it is necessary to prevent negative radii. The main insight of this model, which fits very well the experiments of Portet and Dimova [18], as shown by Figure 5 of [22], is the predominance of the aequous viscosity. Actually even if $\eta_{s}$ is much smaller than the lipid viscosity, for pore radii bigger than $1 \mu \mathrm{m}$, the term $C \eta_{s} r$ plays a crucial role in the pore closure since the membrane thickness $h$ is very small of order $10 \mathrm{~nm}$. The term

$$
\eta_{\mathrm{eff}}(r)=\eta_{\mathrm{m}} h+C r \eta_{s},
$$

can be seen as the effective membrane viscosity, which increases linearly with respect to the pore radius. Ryham et al. pointed out that due to the membrane thinness, the aequous viscosity cannot be neglected, explaining the curvature-driven pore closure. Kroeger et al. used another reasoning based on the electrochemical potential to obtain a similar equation accounting for membrane viscosity (see equation (7) of [10]), keeping the hypothesis of cylindrical pores.

3.2. From one pore to the total pore density equation. From these models, Smoluchowski equation, which is a drift-diffusion equation on the distribution function of pores of radius $r$, gives the pore distribution in the space of radii (see Chapter 1). The very important insight of Krassowska and Neu in the late 90's was to approach Smoluchowski equation by an ordinary differential equation on the total pore density $N_{\mathrm{ep}}$, thanks to a subtle asymptotic analysis [13]. 
Interestingly Kroeger et al. [10] pointed out that both BGS-CW model and DAV-model of pore radius lead to the same ordinary differential equation for the pore density

$$
\frac{d N_{\mathrm{ep}}}{d t}=\alpha e^{V_{\mathrm{m}}^{2} / \mathrm{V}_{\mathrm{ep}}^{2}}\left(1-\frac{N_{\mathrm{ep}}}{N_{o}} e^{-q V_{\mathrm{m}}^{2} / \mathrm{V}_{\mathrm{ep}}^{2}}\right),
$$

where $\mathrm{V}_{\mathrm{ep}}$ is the threshold membrane voltage above which electroporation occurs, $N_{o}$ is the pore density at rest, when $V_{\mathrm{m}}$ equals 0 , and $\alpha$ and $q>1$ are $a d$ hoc parameters.

3.3. Pore radii evolution. In the pore density model, all the pores are created with the same radius, $r_{\mathrm{m}}$. This is due to the averaging and the asymptotic analysis performed to pass from the Smoluchowski equation to the simple differential equation on $N_{\mathrm{ep}}$. In [27, 9], Krassowska et al. add another equation for the pore radius evolution $r_{j}$, for $j=1, \ldots, K$, which has the same basis of equation (20):

$$
\frac{d r_{j}}{d t}=\frac{D}{k_{B} T}\left(a_{p}\left(r_{j}\right) V_{\mathrm{m}}^{2}-2 \pi \gamma+\pi r_{j} \sigma_{\mathrm{eff}}\left(r_{j}\right)+\frac{C_{s}}{r_{j}^{5}}\right),
$$

where $a_{p}$ and $\sigma_{\text {eff }}$ are defined as

$$
a_{p}\left(r_{j}\right)=\frac{F_{\max }}{1+r_{h} /\left(r_{j}+r_{T}\right)}, \quad \sigma_{\text {eff }}\left(r_{j}\right)=2 \sigma^{\prime}-\frac{2 \sigma^{\prime}-\sigma_{0}}{\left(1-\frac{1}{4} \sum_{j=1}^{K} \frac{r_{j}^{2}}{R_{\text {cell }}^{2}}\right)^{2}} .
$$

In [9], the link between $K$ and $N_{\mathrm{ep}}$ is not precisely stated, but it seems that the following equality holds:

$$
K(t)=4 \pi R_{\text {cell }}^{2} N_{\mathrm{ep}}(t) .
$$

3.4. The $\mathrm{KN}-$ model for electroporation current. From the total pore density $N_{\mathrm{ep}}$ and from the description of the pore radii evolution, Krassowska, Neu et al. [13, 27, 9] propose the following nonlinear electroporation current $I_{\mathrm{ep}}$ :

$$
I_{\mathrm{ep}}\left(t, V_{\mathrm{m}}\right)=\sum_{j=1}^{K(t)} i_{\mathrm{ep}}\left(r_{j}, V_{\mathrm{m}}\right)
$$

where $i_{\mathrm{ep}}\left(r_{j}, V_{\mathrm{m}}\right)$ is the current through a single pore of size $r_{j}$. In Krassowska and Filev's paper [9] the current is given by

$$
i_{\mathrm{ep}}\left(r_{j}, V_{\mathrm{m}}\right)=\frac{2 \sigma r_{j}}{1+\delta /\left(2 \pi r_{j}\right)} V_{\mathrm{m}}
$$

which is used in recent works by Weaver et al. [29] and Li and Lin [12].

Note that the way $K(t)$ is computed is still unclear. For numerical purpose, Filev and Krassowska proposed to consider only two kinds of pores: $K_{\text {small }}$ for small pores and $K_{\text {large }}$ large pores. At the begining all the pores are created at the same radius, with a density $N_{\mathrm{ep}}$. Since each node is associated to a surface area $\delta A$, the number of small pores $K_{\text {small }}$ is given by $K_{\mathrm{small}}=\delta A N_{\mathrm{ep}}$ on the nodes of the mesh. Then at any point of the mesh on the membrane, the pore radii evolves, and if the radius increases locally, then the $K_{\text {small }}$ is decreased and $K_{\text {large }}$ increases. One of the drawbacks lies in the fact that the number of pores depend on the mesh, which means that the numerical method is not mathematically consistant: changing the mesh $\mathrm{RR} \mathrm{n}^{\circ} 8940$ 
changes the number of pores passing from $K_{\text {small }}$ to $K_{\text {large }}$. However since the transmembrane voltage value is quite robust with respect to the membrane conductance, as shown in subsection 2.3 of section 2 such a variability should not be troublesome, even though it raises modeling issues.

3.5. Back to the electric equation and drawbacks of the $\mathbf{K N}$-model. In order to link the biophysical model of electroporation to the electric cell model, the Kirchhoff equation (12c) is changed into

$$
\mathrm{C}_{\mathrm{m}} \partial_{t} V_{\mathrm{m}}+\mathrm{S}_{0} V_{\mathrm{m}}+I_{\mathrm{ep}}\left(t, V_{\mathrm{m}}\right)=\left.\sigma_{\mathrm{c}} \partial_{\mathbf{n}} U\right|_{\Gamma^{-}} .
$$

KN-model provides a biophysical explanation of pore formation. The grounds of the model consist of cylindrical pore formed by transmembrane voltage. Such hypothesis has been recently confirmed, at least during the formation process, by optical signal-channel recording in Sengel and Wallace's paper [25], even though more complex phenomena are pointed out such as electropore diffusion, and the resealing has not been addressed in this study. Interestingly, KN-model provides a link between pores of the membrane and global electric potential in the whole cell. From the numerical point of view, the model is not easy to solve because it is non linear and stiff (the dynamic of pore creation is very rapid), therefore accurate numerical methods combined with very fine mesh is needed. The computational cost is quite high, especially for Finite Element Methods, therefore most of the numerical studies focus on bidimensional simulations.

The typical behavior of transmembrane voltage $V_{\mathrm{m}}$ is illustrated by Figure 5 , which comes from Smith et al. [28]. During the first microsecond, the membrane is being charged as a capacitor, but once the transmembrane voltage reaches a threshold value, pores are created, leading to a decrease and then a stabilisation of the transmembrane voltage within $2 \mu \mathrm{s}$. At the equator, the electric field is tangent to the membrane and thus the transmembrane voltage does not increase: no electroporation occurs. At the pole, the electric field is normal and the transmembrane voltage is the highest before electroporation, therefore this is the privileged location for pore formation, as shown in Figure C. 5. Such privileged locations near the poles facing the electrodes have been corroborated qualitatively by the experiments in many studies, in particular from Teissié and Rols' group (see for instance [21, 3] and reference therein), Miklavčič's group [8, 7] and also Vernier's team [30].

3.5.a. KN-model v.s experiments. One of the main drawbacks of the KN-model lies in the fact that it barely corroborate quantitatively the experiments. One can point out here a few experimental observations non accounted for by the KN-model.

- According to [29], the maximum electroporation should be achieved after the first pulse. Patch-clamp experiments on chinese hamster ovary DC3F performed by Wegner et al. exhibits a cumulative effect of the pulses [32] leading to a persistent permeabilization, in addition to the transient permeabilization accounted for by the models (see Figure 6). As mentioned by Wegner et al., such a persistent effect could result from a change in lipid property (such as oxidation), which is not accounted for in the KN-model. Interestingly, patch-clamp measurements of membrane intensity do no exhibit systematically 

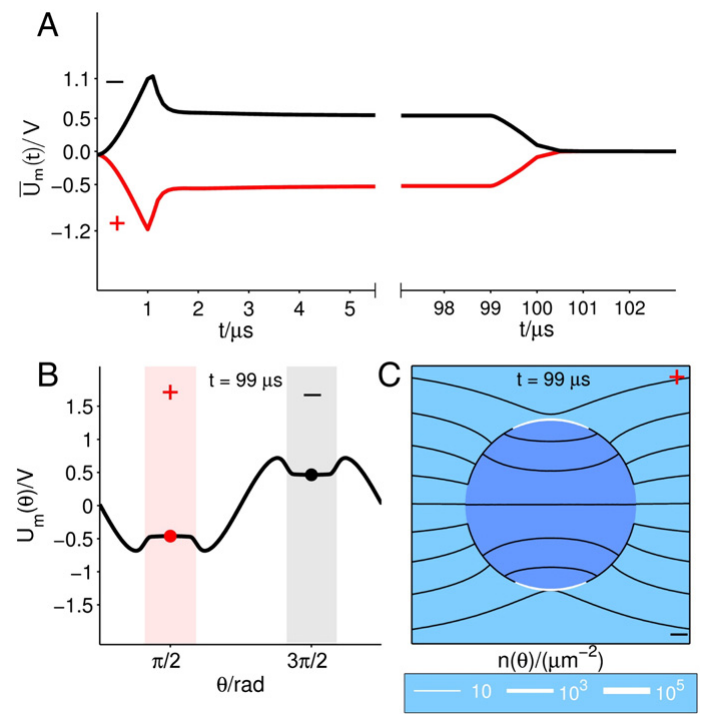

Figure 5. Numerical simulations of KN-model from Figure 3 of [28] (Courtesy of J.C. Weaver). "Electrical and poration response to a $1 \mathrm{kV} / \mathrm{cm}, 100 \mu$ s trapezoidal pulse with $1 \mu$ s rise and fall times. (A) Transmembrane voltage spatially averaged over the polar quadrants, during the initial $5.5 \mu \mathrm{s}$, and at the end of the pulse from 97 to $103 \mu$ s. Red '+' indicates the hyperpolarizing anodic side and black '-' indicates the depolarizing cathodic side. (B) Angular profile of transmembrane voltage at 99 $\mu \mathrm{s}$. Background shaded regions indicate the angular extent of EP around the anodic and cathodic sides of the membrane. Red and black dots mark the location of the anodic and cathodic poles on the membrane, respectively. (C) Equipotentials (black) and pore density, $n(\theta)$ (white), at $99 \mu$ s."

a stabilisation during the pulse unlike KN-model. According to [32, for some patchclamped cells, the current response does not stabilize during the pulse, and moreover the membrane conductance increases with the number of pulses.

- Such cumulative effect is also reported in experiments performed by Pakhomova et al. 14, 2. Whether the pulse repetition rate induces a sensitization or a desensitization is still controversial. Recently Silve et al. reported that high repetition rate of similar pulses is less efficient than the same pulses repeated at a lower rate [26]. These two studies have different explanations of the same phenomenon: the pulse repetition rate has an impact on the electroporation.

- From the modeling point of view, since membrane is a dynamic soft matter (lipid are constantly in motion) it is hard to imagine that no diffusion or motion of pores is involved in the description of pore density. Another important drawback is that saturation of the membrane conductance and pore formation is not accounted for as described e.g. in [32].

The KN-model has been extensively studied in silico during the past decade, providing interesting explanations of electroporation. However the modeling has several drawbacks making the model $\mathrm{RR} \mathrm{n}^{\circ} 8940$ 

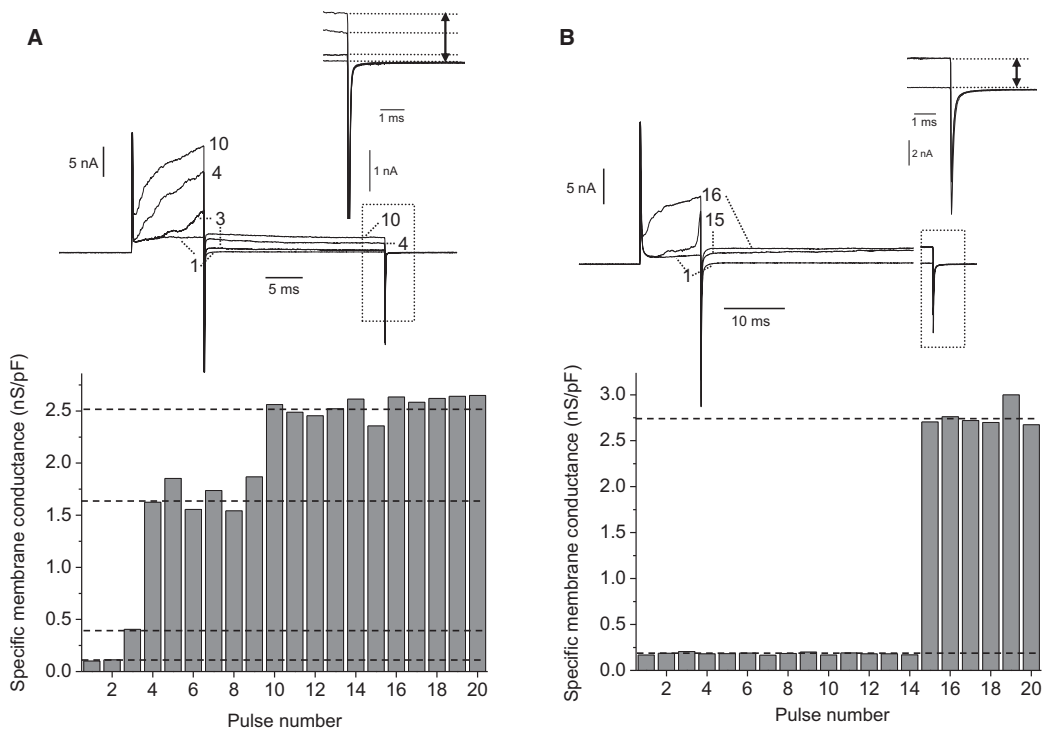

Figure 6. Experimental patch-clamp measurements on DC3F 32. "Two examples of the current response to repetitive application of double-pulse protocol, consisting of a depolarization to $320 \mathrm{mV}$ command voltage for $10 \mathrm{~ms}$ and successive clamp at 80 $\mathrm{mV}$ for 25 or $100 \mathrm{~ms}$ (A and B, upper; note that part of the trace is omitted in B). Subsequently, the membrane was stepped back to $0 \mathrm{mV}$ for $5 \mathrm{~s}$ before the next pulse of the same type was applied. Individual repetitions are superimposed as indicated by numbers. The membrane conductance at the physiological voltage range was calculated from the difference in steady-state current level induced by a voltage step from 80 to $0 \mathrm{mV}$ (redrawn at an enlarged scale in (A) and (B), as indicated by the arrows). Bar graphs ( $\mathrm{A}$ and $\mathrm{B}$, lower) show the conductance levels for the applied sequence of pulses. The conductance increased stepwise (dotted lines), either in several steps (A, pulses 3,4 , and 10) or in one step (B, pulse 15). These two examples were selected to reflect the variability among individual cells."

hardly predictive. The main drawbacks of such modeling lie in the number of non-measurable parameters, and the sensitivity of the results to the parameters which makes the model calibration hardly obtainable. Even though biophysics gives a range for their values, the model is too sensitive to slight modification of many parameters, making hard the parameter calibration with biological data.

\section{Phenomenological models for membrane conductivity}

In order to compare theoretical models and experimental measurements, an alternative to biophysical models is to develop phenomenological models with the fewest parameters to describe the main features of the experiments. The fact that a few parameters are involved facilitate the parameter identification to obtain results that are quantitatively in accordance with the experiments, the drawbacks being the physical justification of the modeling. 
4.1. Biological evidences of electroporation. The phenomenological model of membrane permeabilization proposed by Leguèbe et al. in [11] is based on the following assumptions, which come from experimental observations:

- Permeabilization results from a long-term effect of defects in the membrane related to an alteration of phospholipids in the membrane. Such alterations may be due to the combination of high electric field and water molecules inside the membrane. Actually it has been reported by [6] that electric field changes the phospholipid composition, by altering the lipid property.

- The dynamics of alteration and reconstruction of the membrane are much longer as observed by the experiments [21]. The alteration of the lipids is a physical phenomenon, which occurs as long as pores are present on the membrane and which is persistent after the end of the field pulse. On the contrary, the membrane recovery is a biological phenomenon, which takes time: it happens for minutes after the electric shock.

- Lipids diffuse along the membrane at a speed $\mathrm{d}_{\mathrm{L}}$ around $1 \mu \mathrm{m}^{2} / \mathrm{s}$ [4, which is non negligible compared to the lapse of time between two pulses (usually of the order of 1 second), and therefore this surface diffusion has to be accounted for.

4.2. The two-step process of membrane electroporation. Leguèbe et al. proposed in 11 . a two-step model for membrane electroporation, describing the membrane surface conductance $S_{\mathrm{m}}$ as

$$
S_{\mathrm{m}}(t, \cdot)=\mathrm{S}_{0}+\mathrm{S}_{1} X_{1}(t, \cdot)+\mathrm{S}_{2} X_{2}(t, \cdot),
$$

where $\mathrm{S}_{0}$ is the membrane conductance at rest, $S_{1}$ is the surface conductance of the fully porated membrane, $S_{2}$ is membrane conductance due to the long term effect of electroporation. The nondimension variables $X_{1}$ and $X_{2}$ refer to as the degree of poration during the pulse, and the degree of long term changes in the membrane respectively, and they satisfy the follwing equations:

$$
\begin{array}{ll}
\frac{\partial X_{1}}{\partial t}=\frac{\beta\left(V_{\mathrm{m}}(t, \cdot)\right)-X_{1}\left(t, V_{\mathrm{m}}(t, \cdot)\right)}{\tau_{1}}, & t>0, \\
\frac{\partial X_{2}}{\partial t}-d_{\Gamma} \Delta_{\Gamma} X_{2}=\left[\left(X_{1}-X_{2}\right) / \tau_{2}\right]^{+}, & t>0, \\
\left.X_{1}\right|_{t=0}=X_{1}^{0},\left.\quad X_{2}\right|_{t=0}=X_{2}^{0} . &
\end{array}
$$

where $[\cdot]^{+}$denotes the positive part, while $\tau_{1}$ and $\tau_{2}$ are the characteristic times of "pore" creation and changes in the membrane. The function $\beta$ is a sigmoidal function, for instance

$$
\beta(\lambda)=e^{-\left(\mathrm{V}_{\mathrm{ep}} /\left(\lambda+v_{0}\right)\right)^{2}}, \quad \text { or } \quad \beta(\lambda)=\frac{1+\tanh \left(k _ { \mathrm { ep } } \left(\left(\left|\lambda+v_{0}\right| / V_{\mathrm{ep}}-1\right)\right.\right.}{2} .
$$

The Kirchhoff law is then given by

$$
\mathrm{C}_{\mathrm{m}} \partial_{t} V_{\mathrm{m}}+S_{\mathrm{m}}(t, \cdot) V_{\mathrm{m}}=\left.\sigma_{\mathrm{c}} \partial_{n} U\right|_{\Gamma^{-}} .
$$

Such model involves 6 parameters: $\mathrm{V}_{\mathrm{ep}}$, the two characteristic times $\tau_{1}, \tau_{2}$, the diffusion coefficient $d_{\Gamma}$ and the 2 conductance $S_{1}$ and $S_{2}$.

RR n ${ }^{\circ} 8940$ 

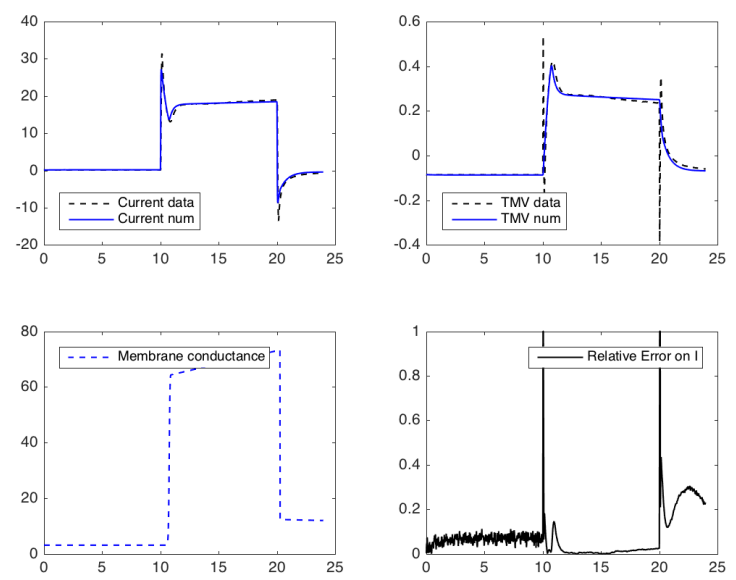

Figure 7. Comparison between simulations and patch-clamp data. The applied voltage is a rectangular function (as described in 32) with a voltage amplitude of $820 \mathrm{mV}$ during 10ms. Thanks to the model, one can compare the currents but also the transmembrane voltage (TMV). The membrane conductance is increasing during the pulse delivery.

4.3. Calibration of the model with patch-clamp experiments. Patch-clamp experiments provide interesting direct measurements of the current that flows across the membrane, for different pulses. Considering patch-clamp experiments on DC3F of [32], it is possible to fit the model parameters with the data. The idea is to use the angular invariance of the command voltage $g$. Assuming that the source potential is applied at the center of the cell -assumed spherical- on a ball of radius $R_{s}$, the flux $\left.\sigma_{\mathrm{c}} \partial_{r} U\right|_{r=R_{\mathrm{c}}}$ reads:

$$
\left.\sigma_{\mathrm{c}} \partial_{r} U\right|_{r=R_{\mathrm{c}}^{-}}=-\left(V_{\mathrm{m}}+g\right) /\left(\frac{R_{\mathrm{c}}}{\sigma_{\mathrm{e}}}+\frac{R_{\mathrm{c}}}{\sigma_{\mathrm{c}}}\left(\frac{R_{\mathrm{c}}}{R_{\mathrm{s}}}-1\right)\right),
$$

and thus the membrane potential $V_{\mathrm{m}}$ satisfies:

$$
I(t):=\mathrm{C}_{\mathrm{m}} \partial_{t} V_{\mathrm{m}}+S_{\mathrm{m}}\left(t, V_{\mathrm{m}}\right)\left(V_{\mathrm{m}}+v_{0}\right)=-\left(V_{\mathrm{m}}+g\right) /\left(\frac{R_{\mathrm{c}}}{\sigma_{\mathrm{e}}}+\frac{R_{\mathrm{c}}}{\sigma_{\mathrm{c}}}\left(\frac{R_{\mathrm{c}}}{R_{\mathrm{s}}}-1\right)\right) .
$$

The feature of the above equation is two-fold: on one hand the patch-clamp technique measures directly the current $I$, and thus one can compare measured and computed intensities. On the other hand the right-hand side of the formula makes it possible to obtain the transmembrane voltage from the measured intensity. The value of the parameters to obtain the currents of Figure 8 are given in Table 1 of Leguèbe et al. [11.

Phenomenological models being designed to be calibrated with the experiments (see Figure 7), it is natural to try to identify parameters that match the data. The key-point being to find a set of parameters such that numerical results fit with as many experiments as possible. Indeed, if different set of parameters are needed for each experiment, it means that the model is not reliable. Interestingly, Figure 8 shows that it is possible to find a set of parameters so that the 

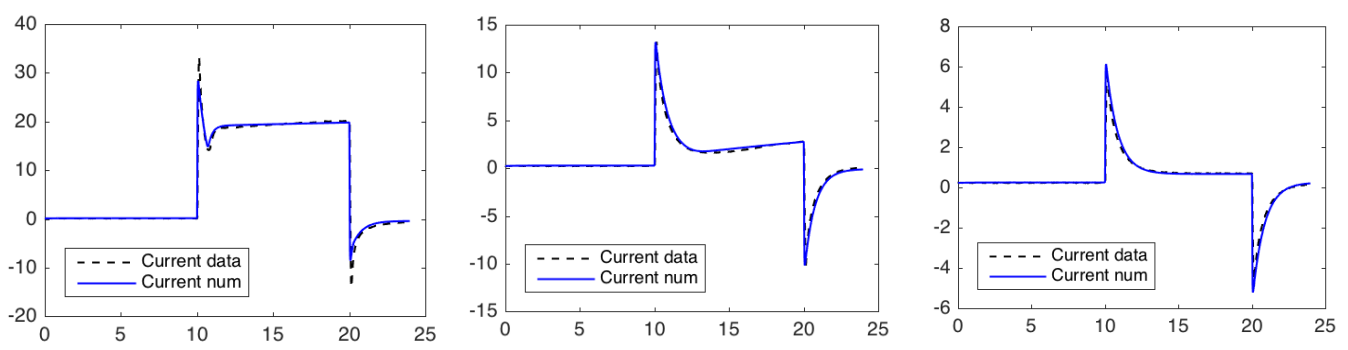

(a) Applied voltage amplitude: $860 \mathrm{mV}$

(b) Applied voltage amplitude: $460 \mathrm{mV}$

(c) Applied voltage amplitude: $140 \mathrm{mV}$

Figure 8. Comparison of the computed and measured currents for different applied voltages to DC3F for a well-chosen set of parameters. Experimental setup is described in 32 . Interestingly, the measured current exhibits a two-step increase, as in the model. Such results cannot be obtained easily with the KN model.

numerical simulations fit quite well with the data. The two functions $X_{1}$ and $X_{2}$ seems necessary to describe the data: the first $X_{1}$ increases very fastly during the pulse, and vanishes at the end of the pulse, while $X_{2}$ increases more slowly, but remains after the pulse. Fitting the KNmodel with these patch-clamp data is proved to be difficult. One reason is the huge number of parameters, which prevents a systematic sensitivity analysis of the model. The two-step process exhibited by the data is hard to obtain with the variable $N_{\mathrm{ep}}$.

\subsection{Biophysical basis of the phenomenological and link with pore radius evolution.}

Equations (33) of the phenomenological model of Leguèbe et al. is very different from the approach of Krassowska et al.. More precisely, equation (33a) can be linked to the pore density equation (25), but the reaction-diffusion (33b) involves surface diffusion of lipids which is not accounted for in Krassowska et al. approach. Even though the physical bases of such reactiondiffusion model is still unclear, it can be related to the phase-ordering kinetics approach, as described in the seminal book of Bray [1]. The concept being to look at the membrane as a twophase medium described thanks to the order-parameter field $\phi$ : the lipid phase, corresponds to the state $\phi=0$, and the water phase to $\phi=1$. The Landau free-energy of the membrane subjected to transmembrane voltage $V_{\mathrm{m}}$ is given by the functional $H(\phi)$ :

$$
H(\phi)=\int_{\Gamma}\left(\frac{1}{2}|\nabla \phi|^{2}+V(\phi)+\frac{1}{2} C_{\mathrm{m}}(\phi) V_{\mathrm{m}}^{2}\right) d s,
$$

where $V$ is a double-well potential with two minima 0 and 1 and $C_{\mathrm{m}}(\phi)$ is the membrane capacitance depending on $\phi$. For instance, Weaver and Chizmadzev proposed $C_{\mathrm{m}}=C_{W} \phi+$ $C_{L}(1-\phi)$ in 31]. From this Landau free-energy, the so-called model A [1] describes the evolution of the order-parameter:

$$
\partial_{t} \phi=-d \frac{\partial H}{\partial \phi}(\phi)
$$

RR $n^{\circ} 8940$ 
leading to the following reaction-diffusion on $\phi$ :

$$
\partial_{t} \phi=d \Delta_{\Gamma} \phi-V^{\prime}(\phi)+\frac{1}{2}\left(C_{W}-C_{L}\right) V_{\mathrm{m}}^{2}
$$

The right-hand side of phenomenological model can be seen as a specific model of the term $V^{\prime}(\phi)-\frac{1}{2}\left(C_{W}-C_{L}\right) V_{\mathrm{m}}^{2}$ in the above equation. The main insight of such approach is to avoid the simplistic assumption of cylindrical pores. It is more general but the equation on single pore can be derived from it. More precisely, the making the assumption that only one cylindrical pore of radius $r$ is present in the membrane supposed to be flat and infinite, then the last equation leads to an equation similar to equation (20), as described in Bray's book [1].

4.5. The role of the surface diffusion. The main novelty of Leguèbe et al. model lies in the surface diffusion, which is numerically difficult to tackle. It is thus important to determine whether or not such surface diffusion plays an important role. Theoretically, the diffusion induces a delay in the spatial response of the membrane, therefore diffusion should appear when several identical pulses at different frequency are applied. In model without surface diffusion, the maximal electroporation occurs after the first pulse, and then the pulse efficiency decreases as reported by Weaver et al. in [29]. In particular the influence of pulse frequency is not accounted for. Surface diffusion induces a complex response, which is a balance between the characteristic time of lipid diffusion and pulse frequency.

In order to investigate the role of the surface diffusion, 3D-simulations of a spherical cell submitted to 10 permeabilizing micropulses $(10 \mu \mathrm{s}, 40 \mathrm{kV} / \mathrm{m})$, with various repetition rates from 1 to $1000 \mathrm{~Hz}$ are presented. The lateral diffusion of the lipids on the membrane is set to

$$
\mathrm{d}_{\mathrm{L}}=10^{-12} \mathrm{~m}^{2} \mathrm{~s}^{-1},
$$

which is in the range of the measured lateral diffusion of the lipids in cell membranes [4].

The average permeabilization $X_{2}$ of the membrane is computed at any simulation time. Figure 9 shows the distribution of $X_{2}$ on the surface of the cell at different instants of the $1 \mathrm{~Hz}$ and $1000 \mathrm{~Hz}$ simulations.

For the case of fast repetition rate, the altered lipids do not have time to be evenly spread on the membrane. Since the next pulse alters the same region as the previous one, and therefore the total quantity of altered lipids is lower than for the $1 \mathrm{~Hz}$ case.

Figure 10 presents the average of $X_{2}$ after each pulse. As expected, the permeabilization is more efficient if enough time is left between pulses to let the lipids diffuse.

These simulations corroborate qualitatively the results of High Voltage/Low Voltage experiments [20, 24] that, within the first seconds after the pulses, show a better permeabilization when the lapse of time between two consecutive pulses is longer.

\section{Concluding Remarks AND PERSPECTives}

In this chapter, biophysical and phenomenological ways to model cell electropermeabilization have been presented, and pros and cons for each approach are discussed. To summarize, biophysical models are more reliable due to their theoretical grounds but they generally involve too many parameters that prevent quantitative comparisons with the biological data. On the 


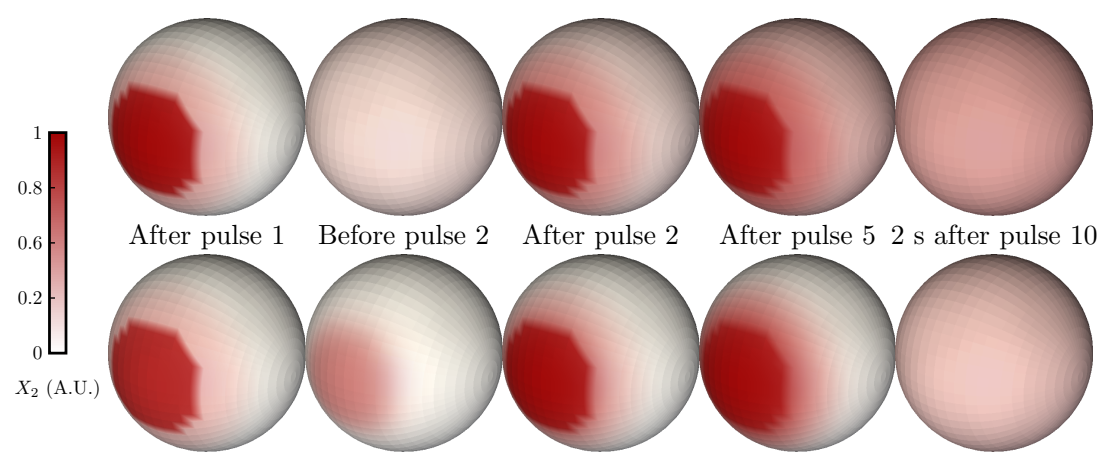

Figure 9. Numerical illustration of the lateral diffusion effect 11. Influence of the pulse frequency on the membrane permeabilization $X_{2}$. The magnitude of each pulse is $40 \mathrm{kV} / \mathrm{m}$ during $10 \mu \mathrm{s}$. 10 pulses are applied on both cells, but the time between pulses is different : 1 second for the top line, 1 millisecond for the bottom line. After 10 pulses, the average of $X_{2}$ is around $8 \times 10^{-8}$ for the $1 \mathrm{~Hz}$ case, and half for the $1000 \mathrm{~Hz}$ case.

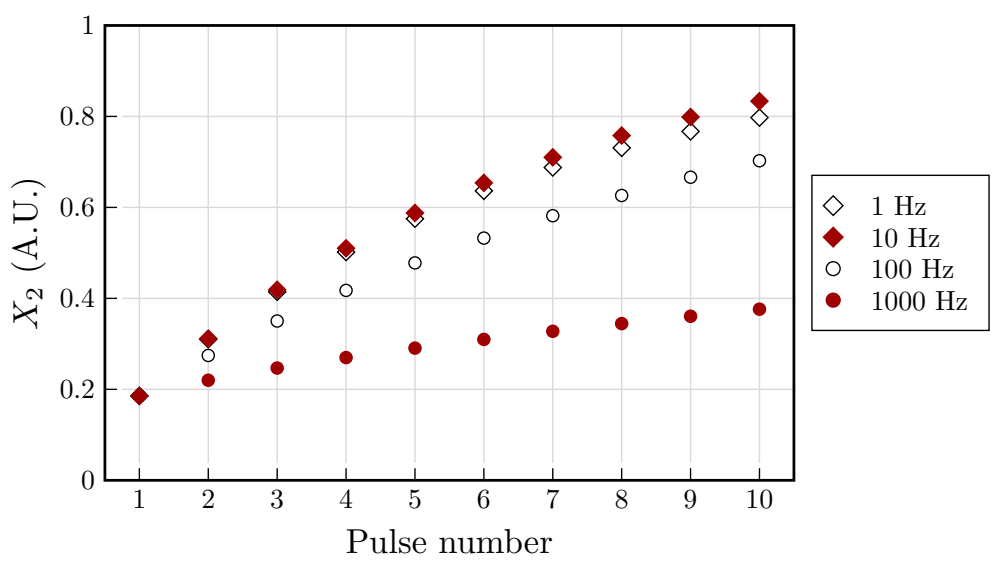

Figure 10. Numerical illustration of the increase of the variable $X_{2}$ [11. Average permeabilization $X_{2}$ on the cell after each of the 10 pulses of Figure 9 for different pulse repetition rates.

other hand, phenomenological models are designed to account for the biological observations, but they suffer from a lack of mechanistic basis. An interesting challenge in cell electroporation modeling would be to enrich the phenomenological modeling with a theoretical grounds, and the phase-ordering kinetics theory could be the appropriate way to tackle this challenges.

The long-term goal would consist in providing a model that can predict quantitatively the degree of cell permeabilization, and the amount of molecules taken up by electroporation. The fitting of the parameters is thus a crucial point, which is common to these perspectives.

$\mathrm{RR} \mathrm{n}^{\circ} 8940$ 


\section{ACKNOWLEDGEMENTS}

This study has been carried out with financial support from the French State, managed by the French National Research Agency (ANR) in the frame of the "Investments for the future", Programme IdEx Bordeaux-CPU (ANR-10-IDEX-03-02).

Numerical simulations presented in this paper were carried out using the PLAFRIM experimental testbed, being developed under the Inria PlaFRIM development action with support from LABRI and IMB and other entities: Conseil Régional d'Aquitaine, FeDER, Université de Bordeaux and CNRS (see https://plafrim.bordeaux.inria.fr/).

C.P. is partly granted by the Plan Cancer project DYNAMO (9749 Inserm), and the Plan Cancer project NUMEP (11099 Inserm). C.P. is also member of the European lab of CNRS, LEA EBAM, on electroporation.

\section{REFERENCES}

[1] A. Bray. Theory of phase-ordering kinetics. Advances in Physics, 51:481-587, 2002.

[2] J. Dermol, O. Pakhomova, A. Pakhomov, and D. Miklavčič. Cell electrosensitization exists only in certain electroporation buffers. PLOS One, 11, 2016.

[3] J. Escoffre, T. Portet, L. Wasungu, J. Teissié, D. Dean, and M. Rols. What is (still not) known of the mechanism by which electroporation mediates gene transfer and expression in cells and tissues. Molecular Biotechnology, 41:286-295, 2009.

[4] P. F. Fahey and W. W. Webb. Lateral diffusion in phospholipid bilayer membranes and multilamellar liquid crystals. Biochemistry, 17(15):3046-3053, 1978.

[5] K. Foster and H. Schwan. Dielectric properties of tissues and biological materials: a critical review. $C R C$ in Biomedical Engineering, 17(1):25-104, 1989.

[6] S. Harakawa, N. Inoue, T. Hori, K. Tochio, T. Kariya, K. Takahashi, F. Doge, H. Suzuki, and H. Nagasawa. Effects of a $50 \mathrm{hz}$ electric field on plasma lipid peroxide level and antioxidant activity in rats. Bioelectromagnetics, 26(7):589-594, 2005.

[7] T. Kotnik, P. Kramar, G. Pucihar, D. Miklavčič, and M. Tarek. Cell membrane electroporation ? part 1: The phenomenon. IEEE Electr. Insul. M., 28, 2012.

[8] T. Kotnik, G. Pucihar, and D. Miklavčič. Induced transmembrane voltage and its correlation with electroporation-mediated molecular transport. J. Membrane Biol., 236, 2010.

[9] W. Krassowska and F. P. D. Modelling electroporation in a single cell. Biophys. J., 92(2):404-4017, 2007.

[10] J. H. Kroeger, D. Vernon, and M. Grant. Curvature-driven pore growth in charged membranes during chargepulse and voltage-clamp experiments. Biophysical journal, 96(3):907-916, Feb. 2009. PMID: 19186129.

[11] M. Leguèbe, A. Silve, L. Mir, and C. Poignard. Conducting and permeable states of cell membrane submitted to high voltage pulses. mathematical and numerical studies validated by the experiments. Journal of Theoretical Biology, 360:83-94, 2014.

[12] J. Li and H. Lin. Numerical simulation of molecular uptake via electroporation. Bioelectrochemistry, 82(1):10$21,2011$.

[13] J. Neu and W. Krassowska. Asymptotic model of electroporation. Physical Review E, 53(3):3471-3482, Mar 1999.

[14] O. Pakhomova, B. Gregory, V. Khorokhorina, A. Bowman, S. Xiao, and A. Pakhomov. Electroporationinduced electrosensitization. Plos One, 6, 2011.

[15] R. Perrussel and C. Poignard. Asymptotic expansion of steady-state potential in a high contrast medium with a thin resistive layer. Applied Mathematics and Computation, pages 48-65, 2013. 
[16] C. Poignard. About the transmembrane voltage potential of a biological cell in time-harmonic regime. ESAIM: Proceedings, 26:162-179, 2009.

[17] C. Poignard and A. Silve. Différence de potentiel induite par un champ électrique sur la membrane d'une cellule biologique. La Revue 3EI, 75:11-20, 2014. http://www.hal.inria.fr/hal-00977590.

[18] T. Portet and R. Dimova. A new method for measuring edge tensions and stability of lipid bilayers: effect of membrane composition. Biophys. J., 84:3263-3273, 2010.

[19] G. Pucihar, T. Kotnik, B. Valič, and D. Miklavčič. Numerical determination of transmembrane voltage induced on irregularly shaped cells. Ann Biomed Eng, 34(4):642-652, Apr 2006.

[20] G. Pucihar, L. Mir, and D. Miklavčič. The effect of pulse repetition frequency on the uptake into electropermeabilized cells in vitro with possible applications in electrochemotherapy. Bioelectrochemistry, 57, 2002.

[21] M. Rols, C. Delteil, M. Golzio, and J. Teissié. Control by ATP and ADP of voltage-induced mammaliancell-membrane permeabilization, gene ransfer and resulting expression. Eur. J. of Biochem., 254(2):382-388, 1998.

[22] R. Ryham, I. Berezovik, and F. Cohen. Aqueous viscosity is the primary source of friction in lipidic pore dynamics. Biophys. J., 101, 2011.

[23] O. Sandre, L. Moreaux, and F. Brochard-Wyart. Dynamics of transient pores in stretched vesicles. Proc. Natl. Acad. Sci, 96:10591-10596, 1999.

[24] S. Šatkauskas, F. André, M. Bureau, D. Scherman, D. Miklavčič, and L. Mir. Electrophoretic component of electric pulses determines the efficacy of in vivo DNA electrotransfer. Hum. Gene Ther., 16(10):1194-1201, 2005.

[25] J. Sengel and M. I. Wallace. Imaging the dynamics of individual pores. PNAS, 113, 2016.

[26] A. Silve, A. Giumerà Brunet, A. Ivorra, and L. Mir. Comparison of the effects of the repetition rate between microsecond and nanosecond pulses: Electropermeabilisation-induced electro-desensitization? Biochimica et Biophysica Acta. General Subjects, 1840, 2014.

[27] K. Smith, J. Neu, and W. Krassowska. Model of creation and evolution of stable electropores for DNA delivery. Biophys. J., 86(5):2813-2826, 2004.

[28] K. Smith, R. Son, T. Gowrishankar, and J. Weaver. Emergence of a large pore subpopulation during electroporating pulses. Bioelectrochemistry, 100:3-10, 2014.

[29] R. Son, T. Gowrishankar, and J. Weaver. Modeling a conventional electroporation pulse train: decreased pore number, cumulative calcium transport and an example of electrosensitization. IEEE Trans. on BioMedical Engineering, 63, 2016.

[30] T. Vernier, Y. Sun, L. Marcu, S. Salemi, C. Craft, and M. Gundersen. Calcium bursts induced by nanosecond electric pulses. Biochemical and Biophysical Research Communications, 310(2):286-295, 2003.

[31] J. Weaver and Y. Chimazdzhev. Theory of electroporation: A review. Bioelectrochemistry and Bioenergetics, 41, 1996.

[32] L. Wegner, W. Frey, and A. Silve. Electroporation of dc-3f cells is a dual process. Biophys. J., 108, 2015. 


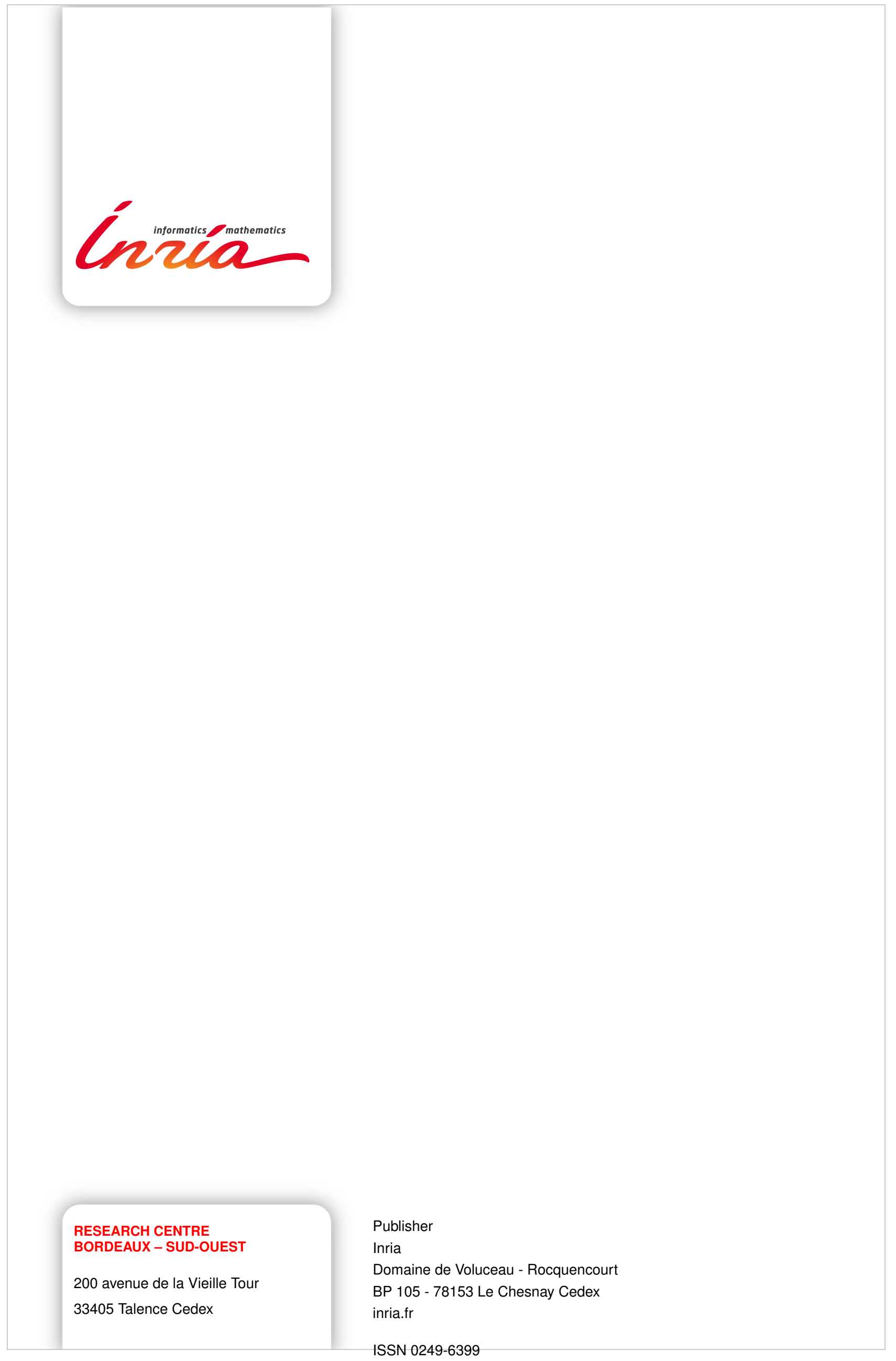

